\title{
Nonlinear theory of scattering by localized potentials in metals
}

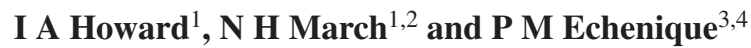 \\ ${ }^{1}$ Department of Physics, University of Antwerp, Groenenborgerlaan 171, B-2020 Antwerp, \\ Belgium \\ ${ }^{2}$ Oxford University, Oxford, UK \\ ${ }^{3}$ Donostia International Physics Center (DIPC), 20018 San Sebastian, Basque Country, Spain \\ ${ }^{4}$ Departamento de Fisica de Materiales and Centro Mixto CSIC-UPV/EHU, Facultad de \\ Quimicas, UPV/EHU, Apartado 1072, 20080, San Sebastian, Spain
}

Received 4 August 2003

Published 29 October 2003

Online at stacks.iop.org/JPhysA/36/11451

\begin{abstract}
In early work, March and Murray gave a perturbation theory of the Dirac density matrix $\gamma\left(\mathbf{r}, \mathbf{r}^{\prime}\right)$ generated by a localized potential $V(\mathbf{r})$ embedded in an initially uniform Fermi gas to all orders in $V(\mathbf{r})$. For potentials sufficiently slowly varying in space, they summed the resulting series for $\mathbf{r}^{\prime}=\mathbf{r}$ to regain the Thomas-Fermi density $\rho(\mathbf{r}) \propto[\mu-V(\mathbf{r})]^{3 / 2}$, with $\mu$ the chemical potential of the Fermi gas. For an admittedly simplistic repulsive central potential $V(\mathbf{r})=|A| \exp (-c r)$, it is first shown here that what amounts to the sum of the March-Murray series for the $s$-wave (only) contribution to the density, namely $\rho_{s}(r, \mu)$, can be obtained in closed form. Furthermore, for specific numerical values of $A$ and $c$ in this exponential potential, the long-range behaviour of $\rho_{s}(r, \mu)$ is related to the zero-potential form of March and Murray, which merely suffers a $\mu$-dependent phase shift. This result is interpreted in relation to the recent high density screening theorem of Zaremba, Nagy and Echenique. A brief discussion of excess electrical resistivity caused by nonlinear scattering in a Fermi gas is added; this now involves an off-diagonal local density of states. Finally, for periodic lattices, contact is made with the quantum-mechanical defect centre models of Koster and Slater (1954 Phys. Rev. 96 1208) and of Beeby (1967 Proc. R. Soc. A 302 113), and also with the semiclassical approximation of Friedel (1954 Adv. Phys. 3 446). In appendices, solvable low-dimensional models are briefly summarized.
\end{abstract}

PACS number: $31.15 . \mathrm{Bs}$

\section{Background and outline}

In early work, March and Murray [1,2] gave a perturbation theory of the Dirac density matrix $\gamma\left(\mathbf{r}, \mathbf{r}^{\prime}\right)$ to all orders in the localized scattering potential $V(\mathbf{r})$ introduced into an originally 
uniform three-dimensional Fermi gas. These authors obtained the electron density $\rho(\mathbf{r})$ as the diagonal limit of their density matrix perturbation theory, and demonstrated that, when $V(\mathbf{r})$ varied sufficiently slowly in coordinate space, their infinite series could be summed to yield the Thomas-Fermi nonlinear relation $\rho(\mathbf{r}) \propto[\mu-V(\mathbf{r})]^{3 / 2}, \mu$ being the chemical potential of the Fermi gas.

Very recent work by Zaremba et al [3], for a potential $\lambda V(\mathbf{r})$ into which a coupling constant $\lambda$ is introduced, has yielded a formally exact expression for $\partial \Delta \rho\left(\mathbf{r}, E_{f}, \lambda\right) / \partial \lambda$ with $E_{f}=\mu$ denoting the Fermi energy of the electron gas, in terms of the Green function $G\left(\mathbf{r}, \mathbf{r}^{\prime}, E\right)$ generated by $\lambda V(\mathbf{r})$, where $\Delta \rho=\rho\left(\mathbf{r}, E_{f}\right)-\rho_{0}$, with $\rho_{0}$ the constant electron density. Of course, it is well known (see, for example, Jones and March [4]) that the Dirac density matrix $\gamma\left(\mathbf{r}, \mathbf{r}^{\prime}, E\right)$ is related to the Green function by

$$
\frac{\partial \gamma\left(\mathbf{r}, \mathbf{r}^{\prime}, E\right)}{\partial E}=\frac{1}{\pi} \operatorname{Im} G\left(\mathbf{r}, \mathbf{r}^{\prime}, E\right)
$$

As set out explicitly below in equation (5.1), the Zaremba, Nagy and Echenique (ZNE) result involves $\operatorname{Im}\left(G^{2}\right)$, and hence the real part of $G$ is also required. In an appendix, $\operatorname{Im} G$ and $\operatorname{Re} G$ are connected via a Kramers-Krönig-type relation, which is given explicitly in the free-electron limit.

Motivated by the ZNE study, we have sought solvable, if somewhat simplistic, models. Thus, in section 2 immediately below, we study analytically a central repulsive exponential potential. Reverting to the early investigation of March and Murray [1], we utilize their treatment of central potentials to study analytically the $s$-wave component $\rho_{s}(\mathbf{r}, E)$ of the density generated by such an exponential potential. Some contact can then be established with the ZNE result. While dealing with localized potentials in a Fermi gas, we shall also treat in section 3 the excess electrical resistivity caused by a central potential $V(\mathbf{r})$. This, as will be emphasized, involves only the imaginary part of the Green function (and now at the Fermi level) displayed already in equation (1.1), and we stress is also intimately related to stopping power (see the review by Echenique et al [5]).

Then, more briefly, we shall consider such localized potentials in periodic metal lattices in section 4. Two exactly solvable quantum-mechanical models fit, rather naturally, into the framework of the present paper. These are due to Koster and Slater [6] and to Beeby [7] respectively. They concern again the Kramers-Krönig-type relation between $\operatorname{Im} G$ and $\operatorname{Re} G$, though in fact they both finish up with the Hilbert transform of the integrated density of states $N_{<}(E)$ as a major component of the displaced electron density due to the perturbation $V(\mathbf{r})$. Section 5 constitutes a summary, plus some proposals for directions in which further studies may prove fruitful. In an appendix, low-dimensional Fermi gases are referred to, again in the context of admittedly simplistic model potentials.

\section{Local density of states for central repulsive exponential potential}

March and Murray [1] gave a partial differential equation for the $l$ th partial wave component, $\rho_{l}(r, E)$, of the total density of electrons at $r$ lying below energy $E$, say $\rho(r, E)$. Putting $l=0$ in their equation (4.12), one can remove their energy integration by considering the local density of $s$-states:

$$
N_{s}(r, E)=\frac{\partial \rho_{s}(r, E)}{\partial E}
$$

to obtain the following third-order ordinary differential equation for $N_{s}(r, E)$ :

$$
\frac{1}{8} \frac{\partial^{3}}{\partial r^{3}}\left(r^{2} N_{s}\right)-\frac{1}{2} \frac{\partial V(r)}{\partial r}\left(r^{2} N_{s}\right)-V(r) \frac{\partial}{\partial r}\left(r^{2} N_{s}\right)+E \frac{\partial}{\partial r}\left(r^{2} N_{s}\right)=0 .
$$


After some investigation, we obtained a general solution of equation (2.2) for the central repulsive exponential potential

$$
V(r)=|A| \exp (-c r)
$$

in terms of Bessel functions $K$ and $I$ as

$$
\begin{aligned}
N_{s}^{\text {general }}(r, E) & =C_{1} \frac{K^{2}\left(2 \mathrm{i} k / c,[8 V(r)]^{1 / 2} / c\right)}{r^{2}}+C_{2} \frac{I^{2}\left(2 \mathrm{i} k / c,[8 V(r)]^{1 / 2} / c\right)}{r^{2}} \\
& +C_{3} \frac{K\left(2 \mathrm{i} k / c,[8 V(r)]^{1 / 2} / c\right) I\left(2 \mathrm{i} k / c,[8 V(r)]^{1 / 2} / c\right)}{r^{2}}
\end{aligned}
$$

where $E=k^{2} / 2$ and now $V(r)$ is written for the exponential potential (2.3). Here, $2 \mathrm{i} k / c$ is the order of the Bessel function. It is a straightforward, if somewhat lengthy, matter to verify by direct substitution of equation (2.4) into the differential equation (2.2) that it is indeed a solution.

Some studies show that $I$ in equation (2.4) is playing the role of an 'irregular' solution of Bessel's equation, and that the physical solution has the form

$$
N_{s}(r, E)=C_{1} \frac{K^{2}\left(2 \mathrm{i} k / c,[8 V(r)]^{1 / 2} / c\right)}{r^{2}} .
$$

As a numerical illustration, figure 1 shows plots of $N_{s}(r, E) / C_{1}$ for three values of $k=\sqrt{2 E}$, and for $V(r)$ in equation (2.3) specified by $|A|=120$ and $c=5$.

\subsection{Comparison of $N_{s}(r, E)$ with free-field form given by March and Murray}

We have noted that the free-field form of $N_{s}(r, E)$ given by March and Murray [1], namely

$$
N_{s}^{(0)}(r, E)=\frac{1}{4 \pi^{2} r^{2} k}[1-\cos (2 k r)]
$$

in the limit of $r$ large, is closely related to the shapes of the three solid curves shown in figure 1. Indeed if a phase shift, $\delta_{0}$ say, is introduced, then the agreement of the free-field $(V(r)=0)$ form (2.6) with the exact result (2.5) is very close, the scale of the local density of states being given correctly when the constant in equation (2.5) is chosen to be inversely proportional to $k$.

\subsection{Relation to the ZNE theorem}

We comment here on the relation of the above findings to the ZNE theorem. First of all, the free-field form $\rho_{s}^{(0)}(r, E)$ is clearly extremely relevant: a consequence of the ZNE result in the limit of large Fermi energy. However, the phase shift $\delta_{0}$ is required to obtain the fully quantitative asymptotic agreement at large $r$ shown in figure 1, when $A$ and $c$ are chosen as indicated.

\section{Excess electrical resistivity created by central potential $V(\mathbf{R})$ in a Fermi gas}

Having discussed at some length an analytical model of the $s$-wave component of the local density of states, we want in this section to summarize how the excess electrical resisitivity, $\Delta R$ say, can be written in terms of the quantities $V(\mathbf{r})$ and the off-diagonal density of states $\partial \gamma\left(\mathbf{r}, \mathbf{r}^{\prime}, E\right) / \partial E$ evaluated at the Fermi energy. One of us [8] took the early work of Huang [9], who expressed $\Delta R$ in terms of the phase shift $\delta_{l}\left(k_{f}\right)$ at the Fermi level for the $l$ th partial wave. A relation of Gerjuoy [10] (see also [11]) was then employed to show that $\Delta R$ was proportional to a force-force correlation function $\langle\mathbf{F} \cdot \mathbf{F}\rangle$, with known proportionality constant, 

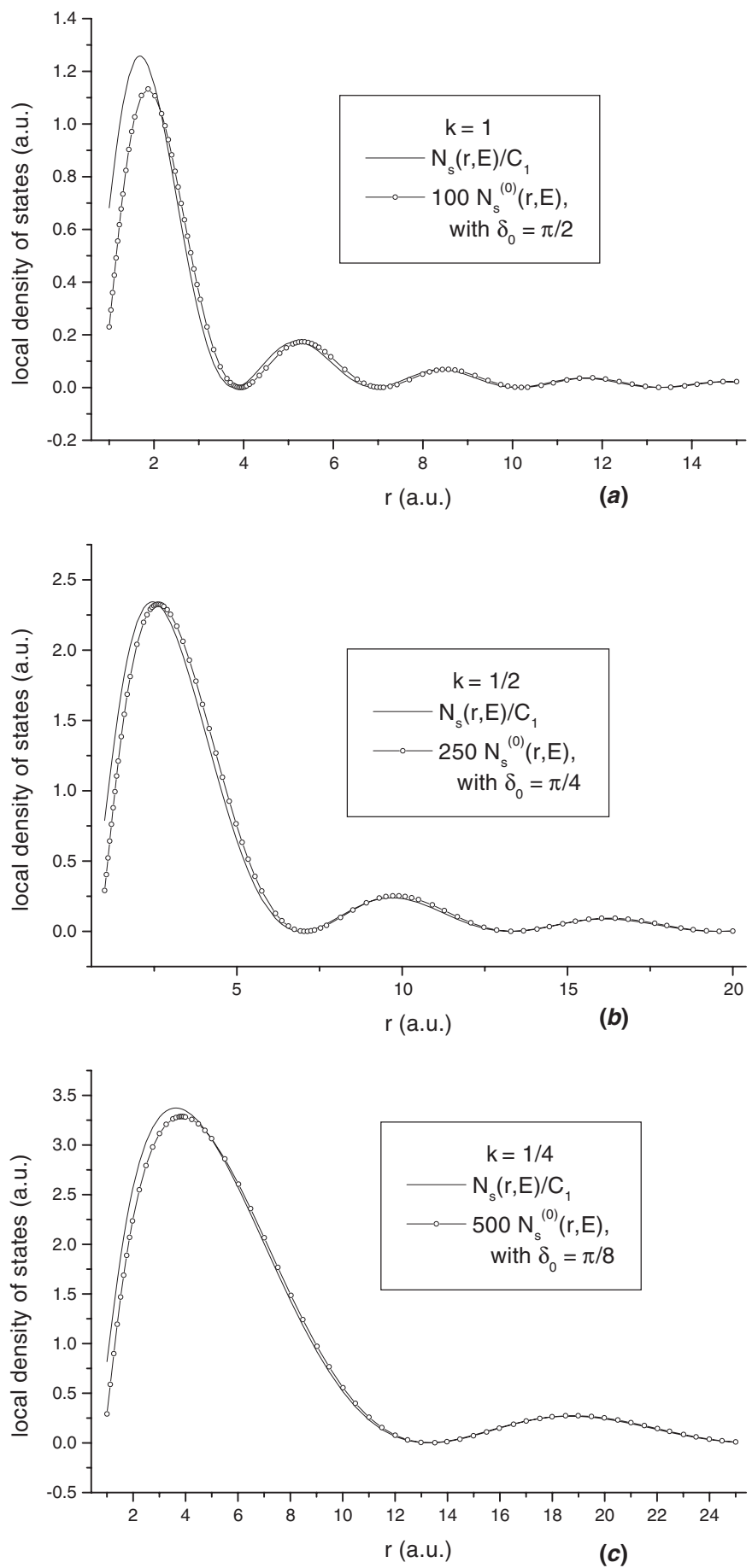

Figure 1. (a)-(c) Local density of $s$-states $N_{s}(r, E) / C_{1}$ for the repulsive exponential potential $V(r)=|A| \exp (-c r)$ with $|A|=120$ and $c=5$, for three values of $k=\sqrt{2 E}$. The free-field form $N_{s}^{(0)}(r, E)=[1-\cos (2 k r)] / 4 \pi^{2} r^{2} k$, in the limit of $r$ large, with a phase shift $\delta_{0}$ and a prefactor as shown, gives the solid curves. 
where $\langle\mathbf{F} \cdot \mathbf{F}\rangle$ is given in terms of the localized (spherical) potential $V(r)$ and the Dirac density matrix $\gamma\left(\mathbf{r}_{1}, \mathbf{r}_{2}, E\right)$ by

$$
\langle\mathbf{F} \cdot \mathbf{F}\rangle=\int \mathrm{d} \mathbf{r}_{1} \mathrm{~d} \mathbf{r}_{2} \frac{\partial V\left(r_{1}\right)}{\partial \mathbf{r}_{1}} \frac{\partial V\left(r_{2}\right)}{\partial \mathbf{r}_{2}}\left[\frac{\partial \gamma\left(\mathbf{r}_{1}, \mathbf{r}_{2}, E\right)}{\partial E}\right]_{E_{f}}^{2} .
$$

Such a formula, but with spherical symmetry on $V(\mathbf{r})$ relaxed, in fact goes back to Rousseau et al [12], who derived it for liquid metals. The major merit there is that the electrical resistivity is immediately obtained to $\mathcal{O}\left(V^{2}\right)$ by putting $\gamma$ equal to its Fermi gas counterpart, namely $\gamma_{0}$ given by

$$
\gamma_{0}\left(\mathbf{r}_{1}, \mathbf{r}_{2}, E_{f}\right)=\frac{k_{f}^{3}}{\pi^{2}} \frac{j_{1}\left(k_{f}\left|\mathbf{r}_{1}-\mathbf{r}_{2}\right|\right)}{k_{f}\left|\mathbf{r}_{1}-\mathbf{r}_{2}\right|} \quad: k_{f}=\left(2 E_{f}\right)^{1 / 2}
$$

where $j_{1}(x)$ is the first-order spherical Bessel function $(\sin x-x \cos x) / x^{2}$.

As a simple example of equation (3.1), let us assume that we can neglect all but $s$-wave scattering to find

$$
\langle\mathbf{F} \cdot \mathbf{F}\rangle_{s-\text { wave only }}=\int \mathrm{d} \mathbf{r}_{1} \mathrm{~d} \mathbf{r}_{2} \frac{\partial V\left(r_{1}\right)}{\partial \mathbf{r}_{1}} \frac{\partial V\left(r_{2}\right)}{\partial \mathbf{r}_{2}}\left[\frac{\partial \gamma_{s}\left(\mathbf{r}_{1}, \mathbf{r}_{2}, E\right)}{\partial E}\right]_{E_{f}}^{2} .
$$

For the exponential potential of section 2, $\partial \gamma_{s} / \partial E$ can be expressed in terms of Bessel functions, to yield the excess resistivity as an explicit integral involving merely Bessel and exponential functions. The scaling properties can then be extracted to yield

$$
\langle\mathbf{F} \cdot \mathbf{F}\rangle_{s \text {-wave only }} \propto \Delta R_{S}\left(|A|, c, E_{f}\right) \text {. }
$$

In the limit of $c$ large, we expect the integration in equation (3.3) to be dominated by small $r_{1}$ and $r_{2}$ and then $\Delta R_{s} \propto\left[|A| \sqrt{E_{f}} c^{-2}\right]^{2}$ is the desired scaling property. But more generally, using an off-diagonal generalization of equation (2.5), we find

$$
\frac{\langle\mathbf{F} \cdot \mathbf{F}\rangle_{s \text {-wave }}}{C_{1}^{2}}=\frac{\sqrt{|A|}}{c} g\left(\frac{\sqrt{E_{f}}}{c}, \frac{\sqrt{|A|}}{c}\right)
$$

reducing the left-hand side of this equation to a function of just two variables $\sqrt{E_{f}} / c$ and $\sqrt{|A|} / c$.

Without the ' $s$-wave only' simplification, it is of interest to stress that the excess resistivity $\Delta R$ only involves the imaginary part of the Green function $G\left(\mathbf{r}_{1}, \mathbf{r}_{2}, E+\mathrm{i} \epsilon\right)$ generated by the potential $V(\mathbf{r})$, in contrast to examples of the local density of states in periodic lattices, to which we now turn.

\section{Two models of defects in a periodic lattice}

\subsection{Koster-Slater model}

Following Koster and Slater [6], we first write the analogue of the Schrödinger equation for the scattered wavefunction $\psi_{\mathbf{k}}(\mathbf{r})$ in terms of the complete set of Bloch wavefunctions $\phi_{m \mathbf{k}}(\mathbf{r})$ of the unperturbed problem, $m$ being a band index and the wave vector $\mathbf{k}$ lying in the first Brillouin zone. The precise form of the integral equation is then

$$
\psi_{\mathbf{k}}(\mathbf{r})=\phi_{m \mathbf{k}}(\mathbf{r})+\sum_{m, \mathbf{k}^{\prime}} \int \frac{\phi_{m \mathbf{k}^{\prime}}^{*}(\mathbf{r}) \phi_{m \mathbf{k}^{\prime}}\left(\mathbf{r}^{\prime}\right) V\left(\mathbf{r}^{\prime}\right) \psi_{\mathbf{k}}\left(\mathbf{r}^{\prime}\right)}{E-E_{m k^{\prime}}+\mathrm{i} \epsilon} \mathrm{d} \mathbf{r}^{\prime} .
$$

Of course, to solve this equation exactly, one must take some model for the defect potential $V(\mathbf{r})$. 
The model of Koster and Slater, though somewhat formal, has the considerable merit that it reduces the scattering problem posed by equation (4.1) to one band, by making an admittedly drastic assumption involving the impurity potential $V(\mathbf{r})$. To formalize their theory, we assume the average of $V(\mathbf{r})$ between Wannier functions $w_{n}\left(\mathbf{r}-\mathbf{R}_{j}\right)$ centred on lattice sites $\mathbf{R}_{j}$ is given by

$$
\int w_{n}\left(\mathbf{r}-\mathbf{R}_{j}\right) V(\mathbf{r}) w_{n^{\prime}}\left(\mathbf{r}-\mathbf{R}_{j}\right) \mathrm{d} \mathbf{r}=V_{n n} \delta_{n n^{\prime}} \delta\left(\mathbf{R}_{j}-\mathbf{R}_{0}\right)
$$

where the impurity is taken to be at the site $\mathbf{R}_{0}$. Then, since the presence of $\delta_{n n^{\prime}}$ in equation (4.2) shows that the impurity potential does not couple different bands one can expand the perturbed wavefunction in equation (4.1) as

$$
\psi_{\mathbf{k}}(\mathbf{r})=\sum_{\mathbf{R}_{j}} u\left(\mathbf{R}_{j}\right) w_{n}\left(\mathbf{r}-\mathbf{R}_{j}\right)
$$

It is then straightforward to show that $u\left(\mathbf{R}_{0}\right)$ is determined by the density of states $n(E)$ in the one band $n$ now remaining:

$$
u\left(\mathbf{R}_{0}\right)=\frac{\exp \left(\mathrm{ik} \cdot \mathbf{R}_{0}\right)}{\left[1-V_{n n} F(E)\right]+\mathrm{i} \pi V_{n n} n(E)} .
$$

The values of $u$ at other lattice sites than the impurity sites can also be computed.

What we want to emphasize here is the central role of the Hilbert transform $F(E)$ of the one-band density of states $n(E)$ of the unperturbed lattice

$$
F(E)=\mathcal{P} \int \frac{n(s)}{E-s} \mathrm{~d} s
$$

where $\mathcal{P}$ denotes the principal value. Dawber and Turner [13] apply the above model to metallic $\mathrm{Cu}$ but we shall not go into further detail here.

\subsection{Beeby model of a lattice vacancy}

We turn to a second 'model' of a vacancy in, say, a metallic crystal such as face-centred-cubic (close-packed) copper. A great simplification, as emphasized by Beeby [7], occurs if we:

(1) start out from a periodic potential modelled as a sum of non-overlapping muffin-tin potentials;

(2) approximate the vacancy by simply removing a muffin-tin potential from the site at which the vacancy is created.

In practice, this is evidently an over-simplistic model, which will eventually need to be refined by (i) allowing for electron redistribution around the vacancy in a self-consistent manner and (ii) accounting for relaxation of the atoms surrounding the vacancy.

Beeby then develops a fully nonlinear scattering theory for the electron density displaced around the vacant site in terms of quantities which, at least in principle, are available from Korringa-Kohn-Rostoker calculations on the perfectly periodic lattice $[14,15]$. For present purposes, we note, for comparison with the Koster-Slater impurity model, a simple example given by Beeby. He derives a now approximate (one-band-like) consequence of his model for the local density of states

$$
\sigma(\mathbf{r}, E)=\frac{\partial}{\partial E} n(\mathbf{r}, E)
$$

where the energy dependence of $\sigma(\mathbf{r}, E)$ is subsumed into

$$
\sigma(\mathbf{r}, E)=f(\mathbf{r}) \frac{n(E)}{F^{2}(E)+\pi^{2} n(E)}
$$


where $f(\mathbf{r})$ is independent of energy. As in the Koster-Slater model, a crucial ingredient is the Hilbert transform $F(E)$ of the density of states $n(E)$ of the host lattice. Here we have the 'fingerprints' of the Kramers-Krönig-like relation between the imaginary and real parts of the Green function emphasized earlier (see also appendix C).

\section{Semiclassical limit of slowly varying potentials $V(\mathbf{R})$}

Let us take as starting point the formally exact expression of Zaremba, Nagy and Echenique [3] (ZNE) for the variation of the 'displaced' electron density $\rho\left(\mathbf{r}, E_{f}\right)-\rho_{0}\left(E_{f}\right) \equiv \Delta \rho\left(\mathbf{r}, E_{f}, \lambda\right)$ in an originally uniform electron gas of density $\rho_{0}$. The ZNE result is explicitly

$$
\frac{\partial \Delta \rho(\mathbf{r}, \lambda)}{\partial \lambda}=\frac{1}{\pi} \int \mathrm{d} \mathbf{r}^{\prime} V\left(\mathbf{r}^{\prime}\right) \int_{E_{f}}^{\infty} \mathrm{d} E \operatorname{Im}\left[G\left(\mathbf{r}, \mathbf{r}^{\prime}, E+\mathrm{i} \epsilon\right) G\left(\mathbf{r}^{\prime}, \mathbf{r}, E+\mathrm{i} \epsilon\right)\right]
$$

for the one-body potential $\lambda V(\mathbf{r})$ inserted into the originally uniform electron gas of density $\rho_{0}$. While $V(\mathbf{r})$ in equation (5.1) is by definition independent of $\lambda, G$ is the Green function generated by switching on the potential $\lambda V(\mathbf{r})$ to the free-electron Green function $G_{0}$. In three dimensions,

$$
G_{0}\left(\mathbf{r}, \mathbf{r}^{\prime}, E+\mathrm{i} \epsilon\right)=\frac{\exp \left(\mathrm{i} \sqrt{2 E}\left|\mathbf{r}-\mathbf{r}^{\prime}\right|\right)}{\left|\mathbf{r}-\mathbf{r}^{\prime}\right|}
$$

One then regains the March-Murray first-order result for the linear response function $F\left(\left|\mathbf{r}-\mathbf{r}^{\prime}\right|, E\right)$ :

$$
\left.\frac{\partial \Delta \rho(\mathbf{r}, \lambda)}{\partial \lambda}\right|_{\lambda=0}=\int \mathrm{d} \mathbf{r}^{\prime} V\left(\mathbf{r}^{\prime}\right) F_{\lambda=0}\left(\left|\mathbf{r}-\mathbf{r}^{\prime}\right|, E\right)
$$

where

$$
F_{\lambda=0}\left(\left|\mathbf{r}-\mathbf{r}^{\prime}\right|, E\right)=\frac{j_{1}\left(2 \sqrt{2 E}\left|\mathbf{r}-\mathbf{r}^{\prime}\right|\right)}{\left|\mathbf{r}-\mathbf{r}^{\prime}\right|^{2}} .
$$

This prompts the observation that the nonlinear response function $F_{\lambda}\left(\mathbf{r}, \mathbf{r}^{\prime}, E\right)$ for the quantity $\partial \Delta \rho(\mathbf{r}, \lambda) / \partial \lambda$ on the left-hand side of equation (5.1) is given by

$$
F_{\lambda}\left(\mathbf{r}, \mathbf{r}^{\prime}, E\right)=\frac{1}{\pi} \int_{E_{f}}^{\infty} \mathrm{d} E \operatorname{Im}\left[G\left(\mathbf{r}, \mathbf{r}^{\prime}, E+\mathrm{i} \epsilon\right) G\left(\mathbf{r}^{\prime}, \mathbf{r}, E+\mathrm{i} \epsilon\right)\right] .
$$

In the semiclassical limit, one must regain (for the unperturbed system as a uniform electron gas in three dimensions)

or

$$
\Delta \rho_{T F}(\mathbf{r}, \lambda)=\frac{8 \pi}{3 h^{3}}(2 m)^{3 / 2}[E-\lambda V(\mathbf{r})]^{3 / 2}
$$

$$
\frac{\partial \Delta \rho_{T F}(\mathbf{r}, \lambda)}{\partial \lambda}=-\frac{4 \pi}{h^{3}}(2 m)^{3 / 2} V(\mathbf{r})[E-\lambda V(\mathbf{r})]^{1 / 2} .
$$

Doing the integral over $\lambda$ between zero and one yields, from equation (5.7):

$$
\begin{aligned}
\Delta \rho_{T F}(\mathbf{r}) & =-\frac{4 \pi}{h^{3}}(2 m)^{3 / 2} V(\mathbf{r}) \int_{0}^{1} \mathrm{~d} \lambda[E-\lambda V(\mathbf{r})]^{1 / 2} \\
& =-\frac{4 \pi}{h^{3}}(2 m)^{3 / 2} V(\mathbf{r}) \int \frac{\mathrm{d} s}{V(\mathbf{r})} s^{1 / 2} \\
& =\frac{8 \pi}{3 h^{3}}(2 m)^{3 / 2}[E-V(\mathbf{r})]^{3 / 2}
\end{aligned}
$$

showing that the nonlinear Thomas-Fermi result thereby follows. 
To conclude this section, we note that the result of Friedel [16] for a periodic $(p)$ lattice with local density $\rho_{p}(\mathbf{r}, E)$ follows similarly from equation (5.1) by semiclassical arguments, and prompted by the TF form (5.8) we can write

$$
\rho_{p}(\mathbf{r}, E)=\rho_{p}(\mathbf{r}, E-V(\mathbf{r}))
$$

Stoddart et al [18] have proposed to extend equation (5.9) by avoiding the appearance of the 'bare' potential $V(\mathbf{r})$, replacing it by a function $g_{1}(\mathbf{r}, E)$ which is approximated by integrals involving $V(\mathbf{r})$ to first order and the off-diagonal generalization $\gamma_{p}\left(\mathbf{r}_{1}, \mathbf{r}_{2}, E\right)$ of $\rho_{p}(\mathbf{r}, E)$. However, we shall not give further details here, the interested reader being referred to the original study [18].

\section{Summary and future directions}

We have been concerned here with nonlinear scattering from a localized potential $V(\mathbf{r})$. The major findings of the present study are dominantly concerned with the central potential $V(|\mathbf{r}|)$ introduced into an initially uniform Fermi gas. In particular, figure 1 shows the electron density for $s$-states only generated by the repulsive exponential potential $V(\mathbf{r})=|A| \exp (-c r)$ for given $A$ and $c$, and for three different values of the Fermi wave number, corresponding to $k=1 / 4,1 / 2$ and 1 respectively. For each case the exact $s$-wave density is shown in the solid curves. The dots in figure 1 represent an 'intuitive' asymptotic form in equation (2.6), in which one merely has the $r$-space dependence of the $s$-wave component of the free Fermi gas corresponding to $V(\mathbf{r})=0$, but now phase-shifted by $\delta_{0}$ as indicated. This form is found numerically to fit the exact $s$-wave densities at large $r$, the scale being correctly determined when the constant in equation (2.6) is chosen to be inversely proportional to $k$.

The $s$-wave density $\rho_{s}(r, k)$ for the exponential potential constitutes an exact summation in terms of Bessel functions of the March-Murray perturbation series when analysed into partial wave components. The corresponding Dirac density matrix can be utilized to calculate the kinetic energy density $t_{s}(r, E)$ relative to the Fermi gas value $t_{0 s}(r, E)$, which is a sum of the Stoddart-March perturbation series [20] when the $s$-wave is extracted.

Some attention has finally been given to treating a localized potential introduced into an originally periodic metallic lattice. The result of ZNE is the starting point here, and the simplest of the findings in section 4 is the case when $V(\mathbf{r})$ varies by but a small fraction of itself over a characteristic de Broglie wavelength for an electron at the Fermi surface. Then the simplest approximation, namely that of Friedel [16], generalizes the Thomas-Fermi approximation to give back the periodic density $\rho_{p}(\mathbf{r}, E)$ of the periodic lattice when $V(\mathbf{r})$ is switched off. In turn, this result is related to the theorem of ZNE. The fully quantum-mechanical defect centre models introduced by Koster and Slater [6] and by Beeby [7] are shown to fit into the general framework discussed here, and this allows a little more insight to be gained as to their final results and particularly to the appearance in both models of the Hilbert transform of the density of states.

As to future directions, two-dimensional electron gases are of considerable current interest for condensed-matter physics. Therefore, in an appendix, the March-Murray approach is reformulated in two dimensions, the exponential potential again affording a useful model, in the plane of the two-dimensional electron assembly. For the future, to treat a point charge, with appropriate screening, both in and above such a two-dimensional Fermi gas, has potential for interesting physical applications, including stopping power [22], referred to already in section 3 . 


\section{Acknowledgments}

IAH wishes to acknowledge support from the IWT-Flemish region. The contribution of NHM to the present study was made during a stay at DIPC in San Sebastian in 2002. It is a pleasure to acknowledge the stimulating atmosphere and the splendid hospitality of the International Center. PME acknowledges support from Hezkuntza Saila, Basque Government and from the Spanish DGICYT (MEC).

\section{Appendix A. Low-dimensional Fermi gases with model localized potentials}

\section{A.1 Two-dimensional models}

In two dimensions, the Fermi gas corresponds to a canonical density matrix $C_{0}\left(\mathbf{r}, \mathbf{r}^{\prime}, \beta\right)$ given by

$$
C_{0}\left(\mathbf{r}, \mathbf{r}^{\prime}, \beta\right)=\frac{1}{a} \sum_{\mathbf{k}} \exp (-\mathrm{ik} \cdot \mathbf{r}) \exp \left(\mathrm{ik} \cdot \mathbf{r}^{\prime}\right) \exp \left(-\beta k^{2} / 2\right)
$$

where $a$ is the normalization area of the wavefunction $\exp (\mathbf{i k} \cdot \mathbf{r})$. Replacing the summation by an integration readily yields

$$
C_{0}\left(\mathbf{r}, \mathbf{r}^{\prime}, \beta\right)=\frac{1}{2 \pi \beta} \exp \left(-\frac{\left|\mathbf{r}-\mathbf{r}^{\prime}\right|^{2}}{2 \beta}\right) .
$$

The Laplace transform with respect to $\beta(\rightarrow E)$ yields the result [21]

$$
\mathcal{L} C_{0}=G_{0}\left(\mathbf{r}, \mathbf{r}^{\prime},-E\right)=K_{0}\left(\sqrt{2 E}\left|\mathbf{r}-\mathbf{r}^{\prime}\right|\right)
$$

where $K_{0}$ is the modified Bessel function.

Hence, changing $E$ to $-E$ in the argument of $K_{0}$ in equation (A.3) yields the off-diagonal local density of states $N_{0}\left(\left|\mathbf{r}-\mathbf{r}^{\prime}\right|, E\right)$ in the two-dimensional Fermi gas, from equation (1.1), which is true in $d$ dimensions. Thus, to first order in a localized potential $V(\mathbf{r})$, we find

$$
G=G_{0}+\int G_{0} V\left(\mathbf{r}^{\prime}\right) G_{0} \mathrm{~d} \mathbf{r}^{\prime}
$$

and hence the change $\Delta N(\mathbf{r}, E)$ in the local density of states is evidently

$$
\begin{aligned}
\Delta N(\mathbf{r}, E) & =-\frac{1}{\pi} \operatorname{Im}\left[G-G_{0}\right] \\
& =-\frac{1}{\pi} \operatorname{Im} \int G_{0} V\left(\mathbf{r}^{\prime}\right) G_{0} \mathrm{~d} \mathbf{r}^{\prime} .
\end{aligned}
$$

Presumably, for free electrons in two dimensions, $N_{0}\left(\left|\mathbf{r}-\mathbf{r}^{\prime}\right|, E\right)$ must become a constant as $\mathbf{r}^{\prime} \rightarrow \mathbf{r}$, since the areal density $\rho_{0} \propto \pi k^{2}=2 \pi E$ and hence

$$
\frac{\partial \rho_{0}}{\partial E}=\text { constant. }
$$

\section{A.2 One-dimensional delta function model}

Finally, we consider a scaled potential $\lambda V(x)$ introduced into an initially uniform onedimensional Fermi gas. Adapting the integral equation form of the Bloch equation satisfied by the canonical density matrix $C$ to one dimension, one readily finds

$C\left(x, x^{\prime}, \beta\right)=C_{0}\left(\left|x-x^{\prime}\right|, \beta\right)-\int_{0}^{\beta} \mathrm{d} \beta_{1} \int_{-\infty}^{\infty} \mathrm{d} x_{1} C_{0}\left(\left|x-x_{1}\right|, \beta-\beta_{1}\right) \lambda V\left(x_{1}\right) C\left(x_{1}, x^{\prime}, \beta_{1}\right)$ 
where the completeness theorem for the one-electron eigenfunctions is now subsumed into equation (A.7).

For the admittedly simple case when

$$
\lambda V(x) \rightarrow \lambda \delta(x)
$$

equation (A.7) becomes

$C\left(x, x^{\prime}, \beta\right)=C_{0}\left(\left|x-x^{\prime}\right|, \beta\right)-\lambda \int_{0}^{\beta} \mathrm{d} \beta_{1} C_{0}\left(|x|, \beta-\beta_{1}\right) C\left(0, x^{\prime}, \beta_{1}\right)$.

Taking the Laplace transform with respect to $\beta$ then yields

$$
\mathcal{L} C\left(x, x^{\prime}, \beta\right)=\mathcal{L} C_{0}\left(\left|x-x^{\prime}\right|, \beta\right)-\lambda \mathcal{L} C_{0}(|x|, \beta) \mathcal{L} C\left(0, x^{\prime}, \beta\right) .
$$

The special case of equation (A.10) corresponding to $x=0$ evidently reads

$$
\mathcal{L} C\left(0, x^{\prime}, \beta\right)+\lambda \mathcal{L} C_{0}(0, \beta) \mathcal{L} C\left(0, x^{\prime}, \beta\right)=\mathcal{L} C_{0}\left(\left|x^{\prime}\right|, \beta\right)
$$

or

$$
\mathcal{L} C\left(0, x^{\prime}, \beta\right)=\frac{\mathcal{L} C_{0}\left(\left|x^{\prime}\right|, \beta\right)}{\left[1+\lambda \mathcal{L} C_{0}(0, \beta)\right]}
$$

Referring to equation (A.10) and using equation (A.12) in the final form, one finds

$$
\mathcal{L} C\left(x, x^{\prime}, \beta\right)=\mathcal{L} C_{0}\left(\left|x-x^{\prime}\right|, \beta\right)-\lambda \frac{\mathcal{L} C_{0}(|x|, \beta) \mathcal{L} C_{0}\left(\left|x^{\prime}\right|, \beta\right)}{\left[1+\lambda \mathcal{L} C_{0}(0, \beta)\right]}
$$

where

$$
C_{0}\left(x, x^{\prime}, \beta\right)=\exp \left[-\frac{\left|x-x^{\prime}\right|^{2}}{2 \beta}\right] /(2 \pi \beta)^{1 / 2} .
$$

Performing next the Laplace inversion of equation (A.13) yields

$$
\begin{aligned}
C\left(x, x^{\prime}, \beta\right)= & \frac{\exp \left[-\left|x-x^{\prime}\right|^{2} / 2 \beta\right]}{(2 \pi \beta)^{1 / 2}} \\
& -\lambda \exp \left(\lambda\left|x-x^{\prime}\right|\right) \exp \left(\lambda^{2} \beta / 2\right) \operatorname{erfc}\left(\lambda \sqrt{\beta / 2}+\left|x-x^{\prime}\right| / \sqrt{2 \beta}\right) \\
& +\frac{\lambda}{2}\left[\operatorname{erfc}\left(\frac{\left|x-x^{\prime}\right|}{\sqrt{2 \beta}}\right)-\operatorname{erfc}\left(\frac{\left(|x|+\left|x^{\prime}\right|\right)}{\sqrt{2 \beta}}\right)\right] \\
& +\frac{\lambda}{2} \exp \left(\lambda^{2} \beta / 2\right) \exp \left[\lambda\left(|x|+\left|x^{\prime}\right|\right)\right] \operatorname{erfc}\left(\lambda \sqrt{\beta / 2}+\left(|x|+\left|x^{\prime}\right|\right) / \sqrt{2 \beta}\right)
\end{aligned}
$$

which, in the context of the ZNE theorem, is interesting in that the $\lambda$-dependence is quite explicit. The diagonal element $x^{\prime}=x$ of equation (A.10) yields the so-called Slater sum $S(x, \beta)$ as

$$
\begin{array}{r}
S(x, \beta)=\frac{1}{(2 \pi \beta)^{1 / 2}}-\lambda \exp \left(\lambda^{2} \beta / 2\right) \operatorname{erfc}(\lambda \sqrt{\beta / 2})+\frac{\lambda}{2}\left[1-\operatorname{erfc}\left(x \sqrt{\frac{2}{\beta}}\right)\right] \\
+\frac{\lambda}{2} \exp \left(\lambda^{2} \beta / 2\right) \exp (2 \lambda|x|) \operatorname{erfc}\left(\lambda \sqrt{\beta / 2}+\left(|x|+\left|x^{\prime}\right|\right) / \sqrt{2 \beta}\right)
\end{array}
$$

so

$$
S(0, \beta)=\frac{1}{(2 \pi \beta)^{1 / 2}}-\frac{\lambda}{2} \exp \left(\lambda^{2} \beta / 2\right) \operatorname{erfc}(\lambda \sqrt{\beta / 2})
$$


Appendix B. Nonlinear $s$-wave phase shift $\delta_{0}(K)$ for an exponential potential

In the body of the text, we gave some emphasis to exact nonlinear properties of the exponential potential, which will be convenient to write in the form

$$
V(r)=-A \exp (-\mu r) \text {. }
$$

Putting $P=r R$, where $R$ is the radial $s$-state wavefunction, one then has

$$
\frac{\mathrm{d}^{2} P}{\mathrm{~d} r^{2}}+\left[k^{2}+A \exp (-\mu r)\right] P=0 .
$$

Making the independent variable $x$, as defined by

$$
x=\frac{2 \sqrt{A}}{\mu} \exp \left(-\frac{\mu r}{2}\right)
$$

equation (B.2) becomes

$$
\frac{\mathrm{d}^{2} P}{\mathrm{~d} x^{2}}+\frac{1}{x} \frac{\mathrm{d} P}{\mathrm{~d} x}+\left(1+\frac{4 k^{2}}{\mu^{2} x^{2}}\right) P=0 .
$$

From [23], the general solution is

$$
P=C J_{2 \mathrm{i} k / \mu}(x)+D J_{-2 i k / \mu}(x) .
$$

The physical solution $P=r R$ must vanish at $r=0$, corresponding from equation (B.3) to $x=2 \sqrt{A} / \mu$. After some manipulation using the asymptotic form of $P$ as $r \rightarrow \infty$, namely

$$
\begin{aligned}
P \sim C\left\{(\exp (-\mu r / 2) \sqrt{A} / \mu)^{2 \mathrm{i} k / \mu} / \Gamma(1+2 \mathrm{i} k / \mu)\right\} \\
+D\left\{(\exp (-\mu r / 2) \sqrt{A} / \mu)^{-2 \mathrm{i} k / \mu} / \Gamma(1-2 \mathrm{i} k / \mu)\right\}
\end{aligned}
$$

the $s$-wave phase shift can be extracted as

$$
\delta_{0}=\arg \left[\Gamma(1+2 \mathrm{i} k / \mu)(\sqrt{A} / \mu)^{-2 \mathrm{i} k / \mu} J_{2 \mathrm{i} k / \mu}(2 \sqrt{A} / \mu)\right] .
$$

This result (B.7) is illustrated in figure 2. It can be seen from equation (B.7) that $\delta_{0}=\delta_{0}(k / \mu, \sqrt{A} / \mu)$, and we have plotted equation (B.7) as a function of $k$ in the figure, for several values of $\sqrt{A} / \mu$ characterizing the exponential potential (B.1).

It is finally of interest to note that the Born (B) approximation to $\delta_{0}(k)$ is given by

$$
\delta_{0}^{B}(k)=-\frac{2 m k}{\hbar^{2}} \int_{0}^{\infty} V(r)\left(\frac{\pi}{2 k r}\right)\left[J_{1 / 2}(k r)\right]^{2} r^{2} \mathrm{~d} r
$$

and this is also plotted in figure 2 for comparison with the smallest value of $\sqrt{A} / \mu$ shown there.

\section{Appendix C. Kramers-Krönig-type relations, and connection between ZNE theorem and force-force correlation formula}

We were motivated by the account in the book by Rickayzen [24] in constructing this appendix involving Kramers-Krönig-like relations. The aim is to show a connection between the ZNE theorem (5.1) and the force-force correlation function (3.1) when $\gamma$ is replaced by the freeelectron limit $\gamma_{0}$. Starting with the latter quantity $\langle\mathbf{F} \cdot \mathbf{F}\rangle$ we have the result that

$$
\frac{\partial \gamma\left(\mathbf{r}_{1}, \mathbf{r}_{2}, E\right)}{\partial E}=\frac{1}{\pi} \operatorname{Im} G\left(\mathbf{r}_{1}, \mathbf{r}_{2}, E\right) .
$$

This, in addition to knowledge of the one-body potential $V(\mathbf{r})$, which of course is required to generate either the Dirac density matrix $\gamma$ or the Green function $G$ in equation (C.1), 


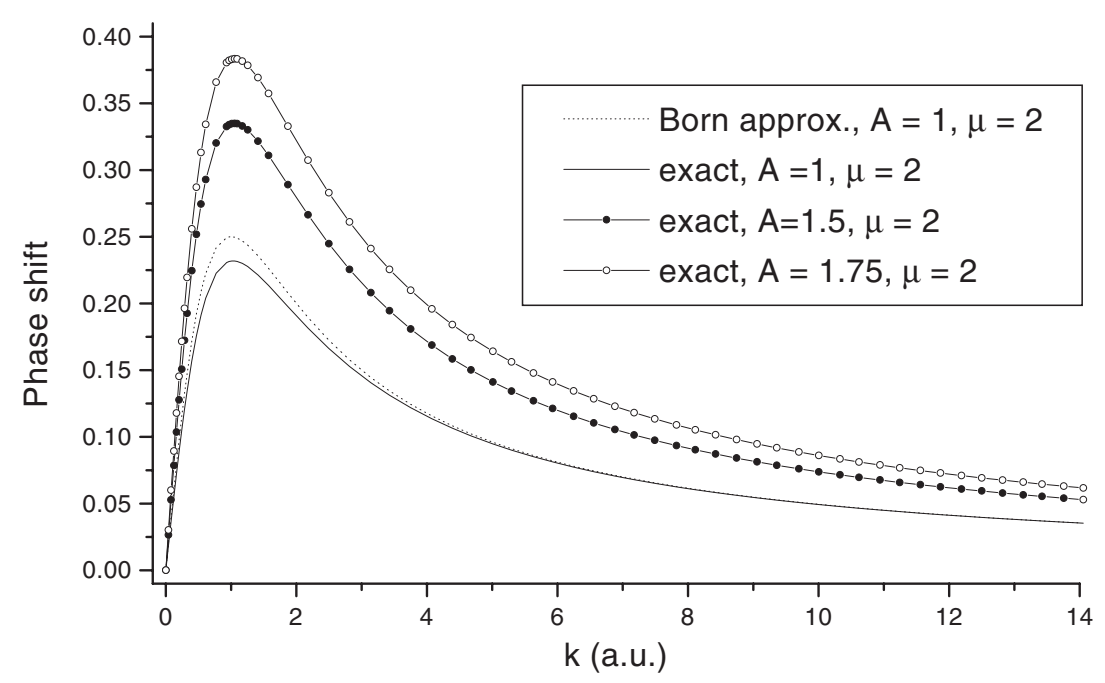

Figure 2. The $s$-wave phase shift $\delta_{0}(k)$ of equation (B.7) for the exponential potential $V(r)=$ $-A \exp (-\mu r)$, for several values of $\sqrt{A} / \mu$; the Born approximation $\delta_{0}^{B}(k)$ of equation (2.6) is shown for comparison for the case $A=1, \mu=2$.

one requires either $(\partial \gamma / \partial E)^{2}$, or from the right-hand side of equation (C.1) alternatively $\left[\operatorname{Im} G\left(\mathbf{r}_{1}, \mathbf{r}_{2}, E\right)\right]^{2}$. This latter quantity is to be contrasted next with what enters the ZNE theorem, namely $\operatorname{Im}\left(G_{0}^{2}\right)$, where $G_{0}$ is now the free-electron limit of $G$ entering equation (C.1). This, of course, even in the limit $V \rightarrow 0$, means that whereas the force-force correlation function is determined, but now only to $\mathcal{O}\left(V^{2}\right)$, by $\operatorname{Im} G_{0}$, one needs both the real and imaginary parts of $G_{0}$ in the ZNE theorem. But, via a Kramers-Krönig-type relation, knowledge of the imaginary part of $G_{0}$ is sufficient to calculate the real part of $G_{0}$ (see equation (C.5))

Let us illustrate specifically the free-electron case by reference to equation (C.1). Then

$$
G_{0}\left(\mathbf{r}_{1}, \mathbf{r}_{2}, E\right)=\frac{\exp (\mathrm{i} \sqrt{2 E} R)}{R}
$$

where $R=\left|\mathbf{r}_{1}-\mathbf{r}_{2}\right|$. Hence

$$
\frac{\partial \gamma\left(\mathbf{r}_{1}, \mathbf{r}_{2}, E\right)}{\partial E}=\frac{1}{\pi} \frac{\sin (\sqrt{2 E} R)}{R} .
$$

Taking the limit $R \rightarrow 0$, we recover the well-known density of states (in three dimensions) proportional to $E^{1 / 2}$ for a free electron gas. With $k=\sqrt{2 E}$, we can show that

$$
\frac{1}{\pi} \mathcal{P} \int_{-\infty}^{\infty} \frac{\sin \left(k^{\prime} R\right)}{R\left(k-k^{\prime}\right)} \mathrm{d} k^{\prime}=\frac{\cos (k R)}{R}
$$

and

$$
\frac{1}{\pi} \mathcal{P} \int_{-\infty}^{\infty} \frac{\cos \left(k^{\prime} R\right)}{R\left(k-k^{\prime}\right)} \mathrm{d} k^{\prime}=-\frac{\sin (k R)}{R}
$$

and thus knowledge of the real part of $G_{0}\left(\mathbf{r}_{1}, \mathbf{r}_{2}, E\right)$ is sufficient to determine the imaginary part, and vice versa. 


\section{References}

[1] March N H and Murray A M 1960 Phys. Rev. 120830

[2] March N H and Murray A M 1961 Proc. R. Soc. A 261119

[3] Zaremba E, Nagy I and Echenique P M 2003 Phys. Rev. Lett. 90 046801-1

[4] Jones W and March N H 1995 Theoretical Solid State Physics (New York: Dover)

[5] Echenique P M, Flores F and Ritchie R H 1990 Solid State Phys. 43229

[6] Koster G F and Slater J C 1954 Phys. Rev. 961208

[7] Beeby J L 1967 Proc. R. Soc. A 302113

[8] March N H 1975 Phil. Mag. 32497

[9] Huang K 1948 Proc. Phys. Soc. 60161

[10] Gerjuoy E 1965 J. Math. Phys. 6993

[11] Gaspari G D and Gyorffy B L 1972 Phys. Rev. Lett. 28801

[12] Rousseau J S, Stoddart J C and March N H 1972 J. Phys. C: Solid State Phys. 5 L175

[13] Dawber P G and Turner R E 1966 Proc. R. Soc. 88217

[14] Korringa J 1947 Physica 13392

[15] Kohn W and Rostoker N 1954 Phys. Rev. 941111

[16] Friedel J 1954 Adv. Phys. 3446

[17] Ziman J M 1964 Adv. Phys. 1389

[18] Stoddart J C, March N H and Stott M J 1969 Phys. Rev. 186683

[19] See also Faber T E 1972 Theory of Liquid Metals (Cambridge: Cambridge University Press)

[20] Stoddart J C and March N H 1967 Proc. R. Soc. A 299270

[21] Abramowitz M and Stegun I A 1970 Handbook of Mathematical Functions (New York: Dover) p 1028

[22] Bergara A, Pitarke J M and Echenique P M 1999 Phys. Rev. B 5910145

[23] Whittaker E T and Watson G N 1927 Modern Analysis (Cambridge: Cambridge University Press) ch xvii

[24] Rickayzen G 1980 Green's Functions and Condensed Matter (New York: Academic) p 41 